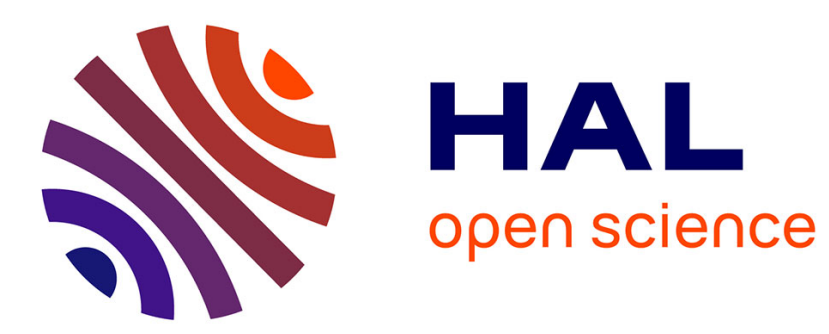

\title{
Convergence of linear finite elements for diffusion equations with measure data
}

Thierry Gallouët, Raphaele Herbin

\section{To cite this version:}

Thierry Gallouët, Raphaele Herbin. Convergence of linear finite elements for diffusion equations with measure data. Comptes rendus de l'Académie des sciences. Série I, Mathématique, 2004, vol. 338, issue 1, pp 81-84. hal-00003437

\section{HAL Id: hal-00003437 \\ https://hal.science/hal-00003437}

Submitted on 1 Dec 2004

HAL is a multi-disciplinary open access archive for the deposit and dissemination of scientific research documents, whether they are published or not. The documents may come from teaching and research institutions in France or abroad, or from public or private research centers.
L'archive ouverte pluridisciplinaire HAL, est destinée au dépôt et à la diffusion de documents scientifiques de niveau recherche, publiés ou non, émanant des établissements d'enseignement et de recherche français ou étrangers, des laboratoires publics ou privés. 


\title{
Convergence of linear finite elements for diffusion equations with measure data
}

\author{
Thierry Gallouët, Raphaèle Herbin
}

Université de Provence, 39 rue Joliot-Curie, 13453 Marseille

E-mail: gallouet, herbin@cmi.univ-mrs.fr

(Reçu le jour mois année, accepté après révision le jour mois année)

Abstract. We show here the convergence of the linear finite element approximate solutions of a diffusion equation to a weak solution, with weak regularity assumptions on the data. (c) 2002 Académie des sciences/Éditions scientifiques et médicales Elsevier SAS

Convergence de la méthode éléments finis $P 1$ pour une équation de diffusion avec second membre mesure.

Résumé. On prouve la convergence des solutions approchées, par la méthode des éléments finis P1, d'une équation de diffusion avec second membre mesure, vers la solution faible de cette équation. (c) 2002 Académie des sciences/Éditions scientifiques et médicales Elsevier SAS

\section{Introduction}

The scope of this work is the discretization by the linear finite element method of diffusion problems on triangular meshes. Let $\Omega$ be a polygonal open subset of $\mathbb{R}^{2}$; the problem under study writes:

$$
\begin{cases}-\Delta u=\mu & \text { in } \Omega \\ u=0 & \text { on } \partial \Omega\end{cases}
$$

with the following hypotheses on the data:

$$
\mu \in M(\Omega),
$$

where $M(\Omega)=(C(\bar{\Omega}))^{\prime}$ is the dual space of $C(\bar{\Omega})$, which may also be identified to the set of bounded measures on $\bar{\Omega}$. In the sequel, we shall consider the usual infinity norm on $C(\bar{\Omega})$, and we shall denote by $\|\cdot\|_{M(\Omega)}$ its dual norm on $M(\Omega)$.

We consider a finite element triangular mesh $\mathcal{M}$ of $\Omega$ (see e.g. [2]), satisfying, for some positive $\zeta$, the following Delaunay and non degeneracy conditions:

\section{Note présentée par Philippe G. CIARLET}

S0764-4442(00)0????-?/FLA

(c) 2002 Académie des sciences/Éditions scientifiques et médicales Elsevier SAS. Tous droits réservés. 


\section{T. Gallouët, R. Herbin}

(i) For any interior edge of $\mathcal{M}$, the sum of the angles facing that edge is less or equal to $\pi-\zeta$.

(ii) For any edge lying on the boundary, the facing angle is less or equal to $\frac{\pi}{2}-\zeta$.

(iii) For any angle $\theta$ of any triangle $T$ of the mesh $\mathcal{M}, \theta \geq \zeta$.

Let $\mathcal{V}$ be the set of interior vertices of $\mathcal{M}$, and let $\phi_{K}$ denote the usual piecewise linear finite element basis function associated with vertex $K$. The usual finite element discretization of (1) with this basis writes:

$$
\sum_{L \in \mathcal{V}} \int_{\Omega} \nabla \phi_{K}(x) \cdot \nabla \phi_{L}(x) u_{L} d x=\int_{\Omega} \phi_{K}(x) d \mu(x), \forall K \in \mathcal{V},
$$

which leads to a linear system of $N$ equations with the $N$ unknowns $u_{L}, L \in \mathcal{V}$, with $N=\operatorname{card}(\mathcal{V})$. The approximate solution is therefore $u_{\mathcal{M}}=\sum_{K \in \mathcal{V}} u_{K} \phi_{K}$.

\section{Convergence of the scheme}

The idea presented here is to compare the finite element scheme on such a mesh with the centered finite volume scheme on the associate Voronoï mesh and to use the results of [5] (or [3], where a more general case is considered) to show the convergence of the scheme.

Indeed, let $\bar{V}$ denotes the set of vertices of the mesh on the whole domain, including the boundary; using the fact that $\sum_{L \in \overline{\mathcal{V}}} \nabla \phi_{L}=0$, the scheme (4) may be written as

$$
\begin{aligned}
& \sum_{L \in \overline{\mathcal{V}}} \tau_{K \mid L}\left(u_{K}-u_{L}\right)=\int_{\Omega} \phi_{K}(x) d \mu(x), \forall K \in \mathcal{V}, \\
& u_{K}=0 \text { if } K \in \overline{\mathcal{V}} \backslash \mathcal{V},
\end{aligned}
$$

with $\tau_{K \mid L}=-\int_{\Omega} \nabla \phi_{K}(x) \cdot \nabla \phi_{L}(x) d x$, for $K \neq L$.

We then construct a dual mesh, denoted $\mathcal{T}$, by considering the control volumes defined by the orthogonal bisectors of the edges of the primal triangular mesh $\mathcal{M}$. In fact, for any $K \in \overline{\mathcal{V}}$, the interior of the control volume of $\mathcal{T}$ associated to $K$ is the set of points whose distance to $K$ is less than its distance to any other vertex of $\overline{\mathcal{V}}$ (for a more detailed description of this so-called Delaunay-Voronoï discretization and its use for covolume methods, we refer to [7] and references therein). The control volumes are also chosen such that they constitute a partition of $\Omega$ (this assumption is important do deal with measures which have some mass on the boundary of some control volumes). The control volume associated with $K$ will also be denoted by $K$. Let us then write the "classical" cell centered finite volume scheme with this mesh (see [5] or [3]):

$$
\begin{aligned}
& \sum_{L \in \overline{\mathcal{V}}} \tau_{K \mid L}\left(u_{K}-u_{L}\right)=\mu(K), \forall K \in \mathcal{V}, \\
& u_{K}=0, K \in \mathcal{V} \backslash \mathcal{V} .
\end{aligned}
$$

We emphasize that the coefficients $\tau_{K \mid L}$ are identical to that of the finite element scheme (5) (which is equivalent to (4)), see e.g. [4], so that the schemes (4) and (6) are the same except for their right-hand-sides. Indeed, if $K, L$ are two distinct vertices of some triangle $T$ of $\mathcal{M}$, then

$$
-\int_{T} \nabla \phi_{K}(x) \cdot \nabla \phi_{L}(x) d x=\frac{1}{2} \operatorname{cotan}\left(\theta_{K, L}\right),
$$

where $\theta_{K, L}$ is the angle of $T$ facing the edge with vertices $K$ and $L$. Hence

$$
-\int_{\Omega} \nabla \phi_{K}(x) \cdot \nabla \phi_{L}(x) d x=\frac{m_{K, L}}{d(K, L)}
$$


where $m_{K, L}$ denotes the distance between the points which are located at the intersection of the orthogonal bisectors of the edges in each of the triangles with vertices $K$ and $L$, and $d(K, L)$ denotes the distance between $K$ and $L$.

Thanks to the construction of this dual mesh, Condition (9) of [5] holds. (More precisely, using the notations of [5], $d_{K, \sigma}=\frac{1}{2} d_{\sigma}$ for any interior edge $\sigma$. If $K$ is a control volume neighbouring the boundary, and if $\sigma$ is an edge of $K$ on the boundary, then $\left.d_{K, \sigma}=d_{\sigma}=0\right)$.

Consider a family of meshes uniformly satisfying Condition (3) for some positive $\zeta$. Theorem 1 of [5] holds for the dual meshes, and therefore, one gets the convergence of the piecewise constant (on the dual cells, that is on the elements of $\mathcal{T}$ ) approximate solution defined by (6), towards the unique weak solution of (1), which is defined by:

$$
\begin{aligned}
& u \in \cap_{1 \leq p<2} W_{0}^{1, p}(\Omega), \\
& \int_{\Omega} \nabla u(x) \cdot \nabla v(x) d x=\int_{\Omega} v(x) d \mu(x), \forall v \in \cup_{q>2} W_{0}^{1, q}(\Omega) .
\end{aligned}
$$

Hence, we shall also obtain the convergence of the solution to (4), that is the finite element approximation of (1), towards the solution of (7). Indeed, the slight difference between (4) and (6) is only due to the right hand side.

THEOREM 2.1. - Let $\Omega$ be an open polygonal subset of $\mathbb{R}^{2}$ and $\mu \in M(\Omega)$. Let $\zeta>0$; for an admissible triangular finite element mesh $\mathcal{M}$ of $\Omega$ satisfying (3), let $u_{\mathcal{N}}=\sum_{K \in \mathcal{V}} u_{K} \phi_{K}$ be the finite element approximation of $(1),\left(\left(u_{L}\right)_{L \in \mathcal{V}}\right.$ is therefore the solution to (4) or (5)). Then $u_{\mathcal{M}}$ tends to $u$ in $L^{p}(\Omega)$, for all $p \in\left[1,+\infty\left[\right.\right.$, and weakly in $W^{1, p}(\Omega)$ for all $p \in[1,2[$, as the mesh size tends to 0 , where $u$ is the solution to (7).

Proof:

The proof of convergence follows that of [5]. We first prove a discrete estimate on the approximate solution (Lemma 1 of [5]), using the test function $\varphi(s)=\int_{0}^{s} \frac{d t}{1+|t|^{\theta}}$, where $\theta>1$. As in [5], we multiply the first equation of scheme (5) by $\varphi\left(u_{K}\right)$ and sum over $K \in \mathcal{V}$. Noting that

$$
\sum_{K \in \mathcal{V}} \int_{\Omega} \phi_{K}(x) d \mu(x) \varphi\left(u_{K}\right) \leq C_{\theta}\|\mu\|_{M(\Omega)} \text {, with } C_{\theta}=\int_{0}^{+\infty} \frac{d t}{1+|t|^{\theta}}<+\infty \text { since } \theta>1,
$$

we see that Inequality (11) of [5] is satisfied, and hence we obtain the estimates in the discrete $W^{1, p}$ norm and in the $L^{p^{*}}$ norm (10) of [5] on the approximate solution $u_{\mathcal{T}}=\sum_{K \in \overline{\mathcal{V}}} u_{K} 1_{K}$ (where $1_{K}$ denotes the characteristic function of $K$ ).

In order to prove that $u_{\mathcal{M}}$ converges to the unique solution of (7), a first possibility is to use the convergence of $u_{\mathcal{T}}$. In this case we use Property (9) given further for the convergence of the right-hand-side and the fact that $u_{\mathcal{T}}-u_{\mathcal{M}}$ converge to 0 in $L^{p}(\Omega)$ for all $p \in[1,+\infty[$ (however this procedure does not yield the convergence of $u_{\mathcal{M}}$ in $W^{1, p}$ weak). A more direct proof is possible, which we now give.

Thanks to the uniform Delaunay condition (3), one remarks that there exists some $C_{1}$, only depending on $\zeta$ such that $m(T) \leq C_{1} m_{\sigma} d_{\sigma}$ if $\sigma$ is an edge of $T$, not lying on the boundary $\left(d_{\sigma}\right.$ is the length of the edge $\sigma$ and $m_{\sigma}$ is the distance between the intersection points of the orthogonal bisectors of triangles $T$ and $S$ if $\sigma$ is common to $T$ and $S$ ). Furthermore, thanks to the non-degeneracy assumption $(i i i)$ in (3), if $K, L$ and $M$ denote the vertices of the triangle $T \in \mathcal{M}$, there exists $C_{2}$ depending only on $\zeta$ such that: $\left|\nabla u_{\mathcal{M}}\right| \leq C_{2} \max \left(\frac{\left|u_{K}-u_{L}\right|}{d(K, L)}, \frac{\left|u_{K}-u_{M}\right|}{d(K, M)}, \frac{\left|u_{L}-u_{M}\right|}{d(L, M)}\right)$ on $T$. Then, the $W^{1, p}$ discrete estimate on $u_{\mathcal{J}}$ leads to a $W_{0}^{1, p}$ estimate on $u_{\mathcal{M}}$. Taking a sequence of meshes with size tending to 0 , then the corresponding approximate solutions $u_{\mathcal{M}}$ tend to some $\mathrm{u}$ in $W^{1, p}$ weak. Let $\psi \in C_{c}^{\infty}(\Omega)$. One multiplies (5) by $\psi(K)$ and sum over $K$ to obtain

$$
\int_{\Omega} \nabla u_{\mathcal{M}}(x) \cdot \nabla \psi_{\mathcal{M}}(x) d x=\sum_{K \in \mathcal{V}} \psi(K) \int_{\Omega} \phi_{K}(x) d \mu(x)=\int_{\Omega} \psi_{\mathcal{M}}(x) d \mu(x),
$$




\section{T. Gallouët, R. Herbin}

where $\psi_{\mathcal{M}}$ is the finite element interpolate of $\psi$ on $\mathcal{M}$. Since $\nabla \psi_{\mathcal{M}}$ tends to $\nabla \psi$ uniformly on $\Omega$ and $\psi_{M}$ tends to $\psi$ uniformly on $\Omega$ as the mesh size tends to 0 , one has:

$$
\int_{\Omega} \nabla u_{\mathcal{M}}(x) \cdot \nabla \psi_{\mathcal{M}}(x) d x \text { tends to } \int_{\Omega} \nabla u(x) \cdot \nabla \psi(x) d x \text { as the mesh size tends to } 0,
$$

and

$$
\int_{\Omega} \psi_{\mathcal{M}}(x) d \mu(x) \text { tends to } \int_{\Omega} \psi d \mu(x) \text { as the mesh size tends to } 0
$$

Passing to the limit in (8), one obtains that $u$ is the solution of (7). This allows us to assert that if the considered meshes satisfy Condition (3), $u_{\mathcal{M}}$ tends to the unique solution of (7) as the mesh size tends to 0 . This concludes the proof of the theorem.

\section{Conclusion}

We proved here the convergence of the piecewise linear finite element scheme for the discretization of a diffusion equation with measure data in two space dimensions.

The above analysis readily extends to the case of the operator $-\operatorname{div} k \nabla$ where $k \in C(\bar{\Omega})$. However, a first important generalization would be to consider any admissible finite element mesh, using the nondegeneracy assumption of the finite element scheme $\rho_{K} \geq C h$ (see [2]) without the Delaunay condition (3). In this case we shall not have $\tau_{K \mid L} \geq 0$ in the finite element scheme (5) (and the discrete maximum principle will not hold). Indeed, in [5], we use the non-negativity of the transmission coefficients $\tau_{K \mid L}$.

Note also that the extension to the three-dimensional case is not straightforward, since there is no easy condition to ensure the equivalence of the finite element and finite volume schemes in the three-dimensional case.

Another generalization would be to deal with general diffusion operators $\operatorname{div} K \nabla u$, where $K$ is a $2 \times 2$ tensor satisfying the usual continuity and coercivity conditions on the associate bilinear form. For a general diffusion operator, it is not possible to interpret the finite element scheme as a finite volume scheme with a two point finite difference approximation of the fluxes on the edges of the mesh. This last property is used in the convergence proof of the finite volume scheme in [5].

For these generalizations, a direct finite element proof is probably the best way to prove convergence. However, a difficulty arises with the fact that if $u_{\mathcal{M}} \in V_{\mathcal{M}}=\operatorname{span}\left\{\phi_{1}, \ldots \phi_{N}\right\}$, where $V_{\mathcal{M}}$ is the finite element space, then the truncations $T_{k} u_{\mathcal{N}}$ (where $T_{k}(s)=\min \{\max \{s,-k\}, k\}$ ) do not in general belong to $V_{\mathcal{M}}$ (see [1] for the use of truncations). Work in this direction is in progress.

\section{References}

[1] L. Boccardo and T. Gallouët, Nonlinear elliptic and parabolic equations involving measure data,. J. Funct. Anal. 87 (1989), 241-273.

[2] P.G. Ciarlet, (1991), Basic error estimates for elliptic problems in: Handbook of Numerical Analysis II (NorthHolland,Amsterdam) 17-352.

[3] J. Droniou, T. Gallouët and R. Herbin,A finite volume scheme for a noncoercive elliptic equation with measure data, accepted for publication in SIAM J. Numer. Anal., 2003.

[4] R. Eymard, T. Gallouët and R. Herbin, Finite Volume Methods, Handbook of Numerical Analysis, Vol. VII, pp. 713-1020. Edited by P.G. Ciarlet and J.L. Lions (North Holland).

[5] T. Gallouët T and R. Herbin, Finite volume methods for diffusion problems and irregular data, in Finite volumes for complex applications, Problems and Perspectives, II, F. Benkhaldoun, M. Hänel and R. Vilsmeier eds, Hermes, $155-162$ (1999).

[6] R. Herbin, An error estimate for a finite volume scheme for a diffusion-convection problem on a triangular mesh, Num. Meth. P.D.E., 11, 165-173.

[7] R.A. Nicolaides, The Covolume Approach to Computing Incompressible Flows, in: Incompressible computational fluid dynamics M.D Gunzburger, R. A. Nicolaides eds, 295-333 (1993). 